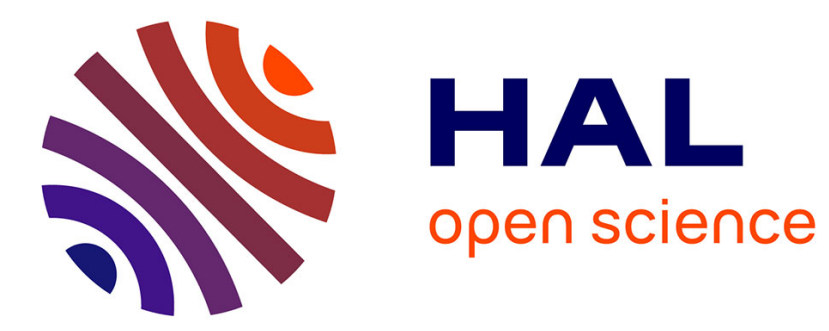

\title{
Acoustic Manipulation of Dense Nanorods in Microgravity
}

Gabriel Dumy, Nathan Jeger-Madiot, Xavier Benoit-Gonin, Thomas Mallouk, Mauricio Hoyos, Jean-Luc Aider

\section{To cite this version:}

Gabriel Dumy, Nathan Jeger-Madiot, Xavier Benoit-Gonin, Thomas Mallouk, Mauricio Hoyos, et al.. Acoustic Manipulation of Dense Nanorods in Microgravity. Microgravity Science and Technology, 2020, 32 (6), pp.1159-1174. 10.1007/s12217-020-09835-7 . hal-03006957

\section{HAL Id: hal-03006957 https://hal.science/hal-03006957}

Submitted on 3 Dec 2020

HAL is a multi-disciplinary open access archive for the deposit and dissemination of scientific research documents, whether they are published or not. The documents may come from teaching and research institutions in France or abroad, or from public or private research centers.
L'archive ouverte pluridisciplinaire HAL, est destinée au dépôt et à la diffusion de documents scientifiques de niveau recherche, publiés ou non, émanant des établissements d'enseignement et de recherche français ou étrangers, des laboratoires publics ou privés. 


\title{
Acoustic manipulation of dense nanorods in microgravity
}

\author{
Gabriel Dumy ${ }^{* \dagger} \quad$ Nathan Jeger-Madiot Xavier Benoit-Gonin \\ Thomas E. Mallouk $k^{\ddagger}$ Mauricio Hoyos ${ }^{\S}$ Jean-Luc Aider $\mathbb{I}$
}

Received: date / Accepted: date

\section{Abstract}

Because the absence of sedimentation in zero-gravity makes the culture and the manipulation of cells or particles challenging, an attractive alternative is to use the Acoustic Radiation Force (ARF) as an artificial "acoustic gravity." To evaluate the potential of this approach we studied the behavior of dense gold nanorods under ARF during a parabolic flight campaign. Using dense objects enhances the effect of gravity on the axial position of the socalled "levitation plane," which is the equilibrium position at which ARF balances gravity in the laboratory. Further, using elongated objects, instead of spherical particles provides information about their spatial orientations in addition to their propulsion observed in standard gravity conditions. Our experiments clearly show a different collective organization and individual behavior of the rods in micro-gravity conditions. First, the axial location of the levitation plane is different in microgravity than in hypergravity: it matches the nodal pressure plane in microgravity while it is much lower than the nodal plane in hypergravity. Our experiments also show a sharp transition from horizontal to axial orientation of the rods axis. The propulsion of the rods also stops when transitioning to micro-gravity. A possible explanation for the sudden change of orientation and stopping of propulsion is

\footnotetext{
*gabriel.dumy@espci.fr

${ }^{\dagger}$ Also at Université Paris Descartes

${ }^{\ddagger}$ University of Pennsylvania

${ }^{\S}$ mauricio.hoyos@espci.fr

$\mathbb{I}_{\text {Corresponding author: jean-luc.aider@espci.fr }}$
} 
the modification of the equilibrium between the axial and transverse components of the ARF. While these experiments show that some phenomena, like the propulsion of nanorods by ARF, may not be applicable in microgravity, they do confirm that acoustic manipulation of particles or cells in microgravity is possible, which paves for the development of many useful techniques for particles or cells manipulation, like cell cultures, during long-term space travel.

\section{Introduction}

Many studies have demonstrated that living organisms, and especially human organisms, do not behave in space as they do on Earth. For instance, it has been shown that heart-rate and arterial pressure change in microgravity $[1,2,3]$. One can also expect significant impacts of long-duration spaceflights or long missions (more than one year) aboard the International Space Station (ISS) on human physiology [4]. If macro-organisms do not behave in microgravity as they do on Earth, this must also be the case for micro-organisms, including human cells. Studying cells in microgravity is not only a challenging and great opportunity, but mandatory to prepare humans for long space missions.

The differences in cell behavior and evolution in microgravity provide us with an opportunity to understand and solve health issue encountered on Earth[5]. However, cell cultures on Earth rely entirely on the sedimentation of the cells toward the substrate of a Petri dish on which they will be grown; this creates a challenge because, in the absence of gravity, cells remain suspended in the culture medium and standard cell culture techniques are not possible. For this reason, cell cultures are usually prepared on Earth, so the cells are already stuck to the substrate prior to being launched toward the space station [6], where their behavior will be studied while still in contact with a solid substrate. While these studies are very interesting and demonstrate that cells behave differently in these conditions than on Earth [7], the ultimate goal is to study the behavior of cells living and growing in the absence of gravity, suspended into a culture medium and without contact with a solid substrate.

One way to study the influence of microgravity on cells - on Earth - is to simulate the microgravity environment using devices called "clinostats," which are $2 \mathrm{D}$ or $3 \mathrm{D}$ rotating devices that achieve weightlessness conditions similar to those experienced in space $[8,9]$.

To create a substrate-free environment on Earth similar to the one 
created in microgravity one can use the Acoustic Radiation Force (ARF) $[10,11]$, which depends on the intrinsic properties (volume, density, and compressibility) of the suspended objects and on the properties of the acoustic field (acoustic frequency and energy), to force particles or cells toward and acoustic pressure node or antinode, where they can be maintained in "acoustic levitation." In this state, cells are no longer distributed randomly in the medium, but concentrated in the so-called "levitation plane" (Fig. 1), which will be discussed thoroughly in the following section. Once in the levitation plane, cells are forced to gather further into a well-defined area of the nodal plane in which they will be trapped due to the radial component of the ARF, which is greatest in the nodal plane [12]. Moreover, once the particles gather in the nodal plane and form a cluster, the Bjerknes force, an indirect close-range inter-particle force induced by particle vibration, helps the cluster to remain stable and packed closely together [13]. This is referred to as an "acoustic trap". Once in the trap, even self-propelled living organisms like bacteria cannot escape the combined ARF and Bjerknes force effects [14]. It is possible to control the shape and geometry of the acoustic field using transducers arrays, leading to controllable equilibrium positions of the levitated objects [15].

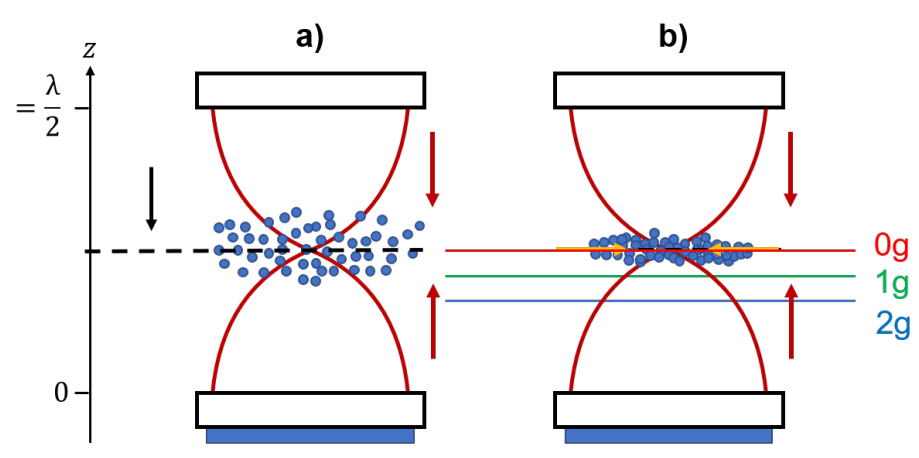

Figure 1: An acoustic pressure node is generated at mid-height within a cavity. Particles with a positive acoustic contrast factor in suspension have migrated toward the acoustic pressure node to form an aggregate that can be maintained in stable acoustic levitation. Usually, one considers the equilibrium aggregate position and the pressure node to be in the same axial position. In reality this position corresponds with the microgravity case. On Earth, the equilibrium position of the acoustic levitation plane is lower than the pressure node, and even lower in hypergravity. 
In a weightless environment, in contrast to the behavior on Earth, the ARF can be used as an artificial gravity [16]. Indeed, in this situation, the acoustic levitation plane on Earth becomes an "acoustic gravity plane"; the particles or cells are forced to gather in the plane of the acoustic pressure node, which can be seen as a local gravity plane. It should then become possible to grow cells inside this plane, as the cells will be forced into confluence by the radial component of the ARF. The micro-gravity environment, together with the absence of mechanical stimulation from the solid substrate, will lead to different cellular behavior than what has previously been observed on Earth and in space - an important point of future study. Unfortunately, studying cellular evolution requires cultures over long times; a minimum of 24 hours is needed to witness significant evolution of the cells' activities, and this is possible only on the ISS.

Nevertheless, there are some fundamental questions that can be tackled in short microgravity experiments with particles, for instance in zero-g flights, before turning to long term experiments on the ISS. Acoustic manipulation techniques are well suited for short experiments thanks to their dynamics and ease of implementation, and have been used to study droplets and bubble oscillations in microgravity for instance [17, 18, 19]. Answering such questions is the objective of this study, led during the October 2018 CNES parabolic flights campaign.

Our main objective is to study the influence of micro-gravity on the acoustic manipulation of particles. On Earth, as previously discussed, particles gather in the levitation plane, which is theoretically considered to be close to the acoustic pressure node plane. This assumption is not reflective of reality, as the actual axial position of the levitation plane is the equilibrium position between gravity and the axial component of the ARF, as shown in Fig. 1. Because using dense metallic particles enhances the difference in this levitation plane position, we used case gold particles in these experiments.

Another interesting question is how the axial location of the levitation plane influences the spatial organization of objects. In order to have a clearer view of the objects' individual orientations, we used elongated particles, specifically gold nanorods.

One very instructive feature of gold nanorods is that, unlike passive spherical particles, they become acoustically-propelled "artificial swimmers" once inside the acoustic levitation plane [20]. This phenomenon has been called "self-acoustophoresis" and could be used, for instance, in drug-delivery applications [21]. Therefore it is important to investigate whether selfacoustophoresis is possible in microgravity. Moreover, microgravity experiments may help in understanding the origins of self-acoustophoresis. Indeed, 
this phenomenon is only observed with dense objects, and has no effect for instance on silicon-carbide fibers[22], suggesting a role of gravity in the process, even if the only plausible theoretical explanations so far suggest that fluid streaming around the oscillating elongated rods may be responsible for this acoustic propulsion $[23,24]$. It has been demonstrated that acoustic streaming propulsion could be generated through bubble oscillation, that have a much larger deformation capacity than the metallic rods used in this study $[25,26]$.

To summarize, the objectives of these experiments are three-fold:

- first, to evaluate for the first time the real position of the acoustic levitation plane using dense particles;

- second, to evaluate the influence of the ARF axial and radial components on the spatial organization of elongated objects;

- third, to check whether the modification of the location of the acoustic levitation plane has an effect on the self-acoustophoresis of dense nanorods.

In the following, we first recall the theoretical basis of acoustic levitation and discuss the axial position of the levitation plane, depending on the particles' density. Then, we present the experimental setup, particularly its adaptation to the constraints of parabolic flights, which allows us to observe micrometric objects in a challenging noisy environment. Finally, we present our observations, in which we discuss thoroughly several of the classic hypotheses frequently used in acoustofluidics and, in particular, in self-acoustophoresis theoretical background. We confirm that it is possible to handle efficiently large numbers of particles or cells in microgravity, but it appears that it may be impossible to use self-acoustophoresis in zero-g, as the propulsion mechanism seems linked to the position of the levitation plane.

\section{Theoretical considerations}

\subsection{Discussion about the actual axial position of the acoustic levitation plane for dense objects}

Fig. 1 depicts the fundamental mode of an ultrasonic resonator composed of two infinite parallel plates. Fulfilling the condition $h=\lambda_{a c} / 2$, where $h$ is the height of the cavity or micro-channel and $\lambda_{a c}$ is the acoustic wavelength, 
generates one node at mid-height of the cavity where the acoustic pressure $p_{a c}=0$ and two pressure anti-nodes on the walls of the cavity. In this case, the suspended particles can be driven by the ARF towards the node or the antinodes, depending on their acoustic properties.

The acoustic radiation force has been theoretically derived for spherical particles, first by King [10] (rigid and incompressible spheres), improved by Yosioka [11] (compressible spheres), then advanced even further by Gor'kov [27] (ideal fluid) and by Doinikov and Bruus [28, 29] (viscous fluid). In the following we will consider the model proposed by Yosioka, where the $z$ axis is aligned with the direction of the acoustic wave, as shown in Fig. 1, and which defines the axial component of the ARF as:

$$
\boldsymbol{F}_{\text {rad }}^{\text {axial }}=4 \pi a^{3} F_{Y} k_{a c}\left\langle E_{a c}\right\rangle \sin \left(2 k_{a c} z\right) \boldsymbol{e}_{z}
$$

where $\langle-\rangle$ denotes time averaging; $a$ is the particle radius; $E_{a c}$ is the acoustic energy density; $k_{a c}=\frac{2 \pi}{\lambda_{a c}}=\frac{2 \pi f_{a c}}{c_{0}}$ is the wave number of the acoustic plane wave of frequency $f_{a c}$; and $z$ is the axial (or vertical) position of the particle, where $z=0$ represents the bottom of the channel and $z=h$ represents the top.

The acoustic contrast factor $F_{Y}$ of a given particle of density $\rho_{p}$ in a medium of density $\rho_{0}$, exposed to an acoustic wave propagating at $c_{0}$ in the medium and at $c_{p}$ in the particle, is defined as:

$$
F_{Y}=\frac{1+\frac{2}{3}\left(1-\rho_{0} / \rho_{p}\right)}{2+\rho_{0} / \rho_{p}}-\frac{\rho_{0} c_{0}^{2}}{3 \rho_{p} c_{p}^{2}}
$$

In general, this expression is a positive numerical value for any object that is denser and less compressible than the suspending fluid, like polystyrene or silica particles in water or cells in plasma. When $F_{Y}$ is positive, the particles are forced to move toward the pressure nodes of the acoustic field, where they gather to form aggregates. $F_{Y}$ is negative for objects that are lighter and softer than the suspending fluid, like air bubbles or lipid droplets in water. In this case, the objects move toward the pressure anti-nodes (i.e. velocity nodes). This phenomenon is often referred to as "acoustophoresis" or acoustic levitation, as it opposes gravity and buoyancy and can keep one or multiple particles in a stable equilibrium position.

Once the particles have reached the levitation plane, the axial position of the aggregate they form depends on the balance between gravity and buoyancy forces acting upon them. The location of the aggregate at mid-height of the cavity is an asymptotic solution of Eq. 1 that assumes infinite acoustic 
energy and zero gravity or totally buoyancy-neutral particles. It is interesting, then, to study the behavior of particle suspension under various gravity regimes, since the acoustic force can be considered an artificial gravity [16] and can be exploited in zero-g environments to provide local acceleration to suspensions when needed (for example, for cell manipulation or cultures in space).

In the framework defined in Fig. 1, we have the following force balance at equilibrium:

$$
\boldsymbol{F}_{a c}+\boldsymbol{F}_{\text {buoy }}+\boldsymbol{F}_{\text {grav }}=\mathbf{0}
$$

leading to the following expression for the normalized equilibrium position using Eq. 1:

$$
\frac{z_{e q}}{h}=\frac{1}{2 \pi} \times \arcsin \left[\frac{g\left(\rho_{f}-\rho_{p}\right)}{3 k_{a c} F_{Y}\left\langle E_{a c}\right\rangle}\right]
$$

The final equilibrium position of gold or polystyrene particles as a function of the acoustic energy for three different gravitational fields intensities is plotted on Fig. 2. As shown, the deviation from the theoretical nodal position is small for polystyrene particles, while it is much larger for gold particles. A vertical asymptotic line denotes a settling of the aggregate. In both standard gravity and hypergravity, a minimal acoustic energy level is needed to trigger the levitation. However, significantly more acoustic energy is required for the levitation plane to reach the actual pressure node. Thus, under everyday laboratory conditions, the acoustically propelled metallic nanocylinders presented in Wang et al. [20] are actually observed to be in a vertical position that is considerably lower than the real geometric pressure node of the acoustic field. This point has never been considered or observed in previous studies, despite its potential importance as it suggests a different balance between the axial and radial components of the ARF.

\subsection{Discussion of the behavior of elongated objects}

Apart from the modification of the axial position of the levitation plane when dealing with dense particles, it has also been shown that elongated dense particles do not behave the same way as spherical particles. Instead of aggregating passively inside the levitation plane, nanorods are suddenly propelled and start moving randomly inside the levitation plane [30] with various trajectories [31]. Further, the directed chirality of the particles can

be used to influence proper rotation under acoustic actuation [32]. It is very 

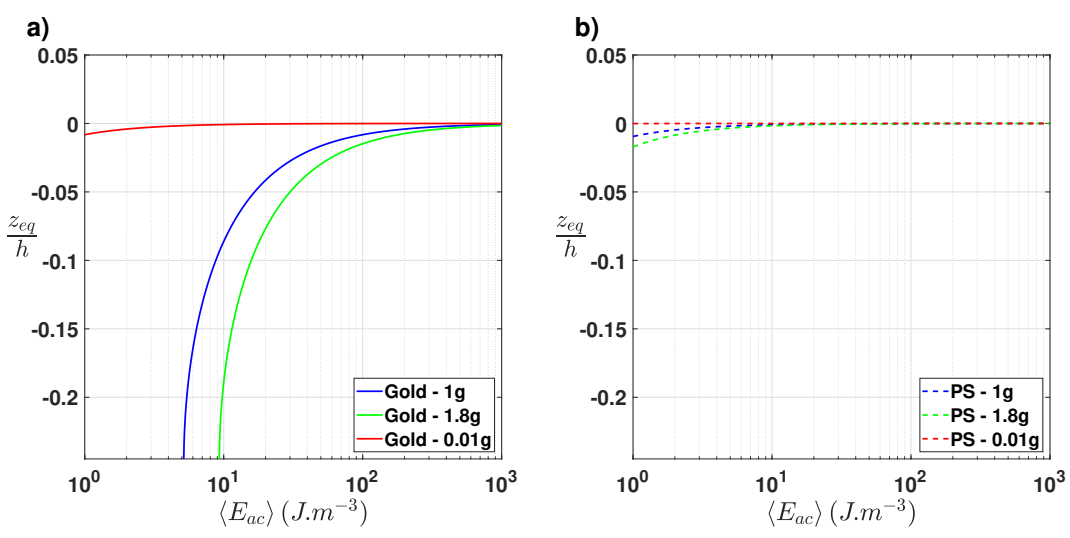

Figure 2: a) Evolution of the normalized position of equilibrium for gold particles, as a function of the acoustic energy carried by the acoustic field at $f_{a c}=3.65 \mathrm{MHz}$, for the three acceleration regimes accessible during microgravity flights. b) Same evolution for polystyrene particles. The influence of gravity on the location of the levitation plane is much stronger for gold particles than for polystyrene particles.

interesting, then, to try to evaluate the influence of the actual location of the levitation plane on the autonomous propulsion of the metallic nanorods.

Another benefit of using elongated particles is that the orientations of their long axes can be evaluated. This point may have been overlooked in most of the previous studies. Indeed, previously reported observations show that nanorods are propelled inside the levitation plane, and that their long axes are parallel to the levitation plane and generally aligned in the same direction in which they are being propelled. Fig. 3 shows a typical snapshot of acoustically propelled nanorods observed in the laboratory for a given set of acoustic parameters. One can see that the nanorods long axes are always inside the levitation plane as sketched in Fig. 4b) and their orientation does not have a random distribution (Fig. 4a)). It makes sense from a hydrodynamic point of view to have the long axis aligned with the propulsion direction, but the explanation for the long axis alignment with the nodal plane was never clearly established.

The orientation of the long axis of elongated objects can also be used as a proxy value for the equilibrium between the forces applied to these objects. If the location of the levitation plane is modified by gravity, then the forces applied to the particles will also be altered, leading to a change in orientation. This point will be discussed in a following section. 


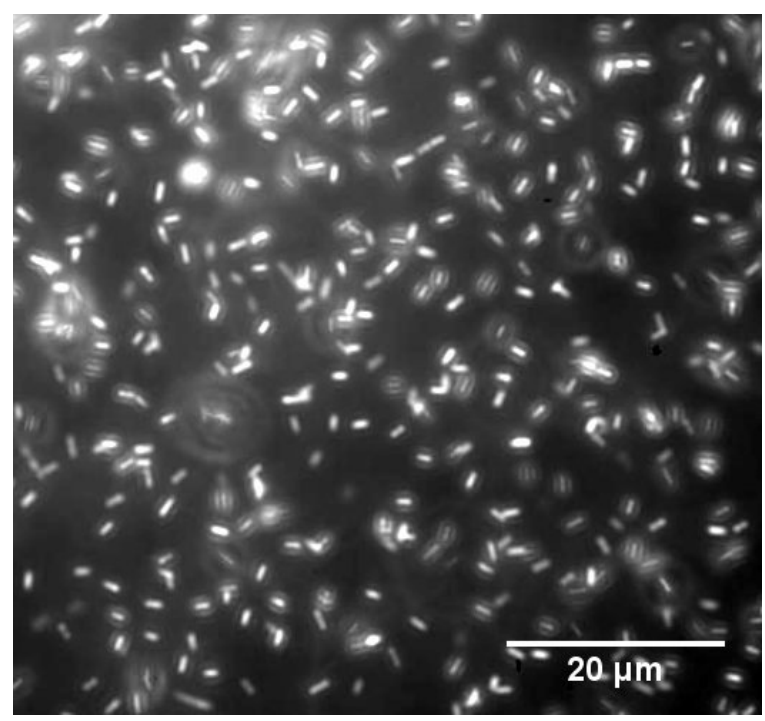

Figure 3: Snapshot of acoustically-propelled gold nanorods observed in the levitation plane with dark-field microscopy, in standard laboratory conditions $\left(f_{a c}=1.83 \mathrm{MHz}, U_{p p}=10 \mathrm{~V}\right)$. Movie online.

\section{Experimental set-up}

\subsection{Acoustic resonator for zero-g flights}

Designing a resonant acoustic cavity is relatively straightforward. Indeed, all that is needed to trap a fraction of the acoustic energy injected by the acoustic source is a cavity with hard enough boundaries and an approximate matching of the acoustic wavelength in at least one dimension. Designing a good resonant cavity - one that allows us to achieve specific objectives - is challenging. Our cavity must allow us to identify the locations of the principal and secondary nodes generated by the acoustic field. And, in order to allow us to manipulate a broad range of objects, particularly dense gold and platinum nanorods, it must be designed so as to maximize the amplitude of the ARF.

Another challenge in designing a good resonant acoustic cavity is associated with the constraints of the zero-z flights. The cavity must be sealed so that the fluid medium does not leak during flight. The design must also allow us to swiftly change both the fluid medium and the nanorods or particles used between each set of parabolas (usually there is a 10 minute break after five parabola). In addition, we used a dedicated, closed micro-channel 


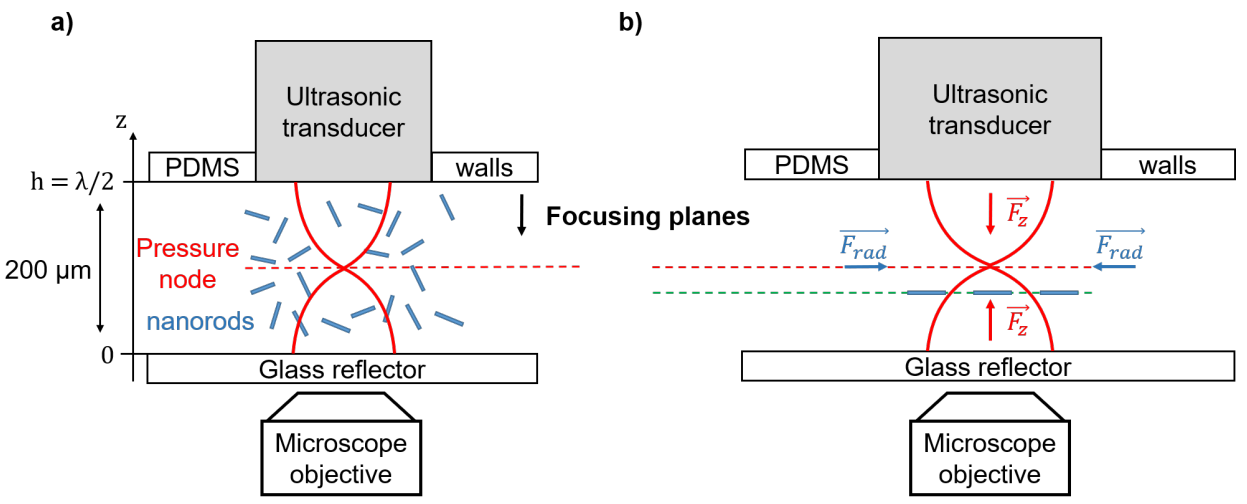

Figure 4: a) Sketch of the acoustic resonant cavity. The cavity height is $200 \mu \mathrm{m}$, resulting in an acoustic resonant frequency $f_{a c}=3.65 \mathrm{MHz}$. The cavity's upper wall is directly the quarter-wave layer of a packaged transducer, embedded in PDMS walls. b) When the ultrasounds are turned on the nanorods gather at the equilibrium levitation plane and form a swarming cluster. The equilibrium levitation plane is lower than the nodal plane due to the effects of gravity, and the distance from the nodal plane is larger for high-density nanorods, like the gold ones used in this study. In microgravity, nanorods are expected to gather in the nodal plane.

built in PDMS (polydimethylsiloxan) bonded to a glass microscope slide, which allows a better matching of the frequency, a larger acoustic force, and a well-defined acoustic focusing area. As described in Fig. 4, the upper-wall of the cavity is the quarter-wave length layer of the ultrasonic transducer (Signal Processing TR0408SS 4MHz packaged transducer), which is in direct contact with the fluid medium. $1 \mathrm{~mm}$ glass microscope slide serves as the reflecting layer. The single node resonance for a cavity height $h=200 \mu \mathrm{m}$ is obtained with an acoustic resonant frequency $f_{a c}=3.65 \mathrm{MHz}$. The transducer is actuated by a TiePie wave-generator, controlled electronically.

\subsection{Microscopy}

In order to simultaneously observe and manipulate our samples, we used an inverted microscope coupled with an $X, Y, Z$ motorized stage, based on a LaVision Flowmaster MITAS setup, and manually controlled. The rods are observed using an Olympus x10 objective and a Hamamatsu OrcaFlash $4.0 \mathrm{v} 1$ camera, directly streaming the images onto the controlling computer. The samples are illuminated by a pe4000 CoolLed light source connected to 
the microscope through an optical fiber (Fig. 5).

\subsection{Experiment control}

Injection of samples and buffer was done by a KD Scientific Legato 100 Series syringe pump, also controlled by the computer (Fig. 5). The tubing and connections were prepared on the ground prior to the flights. Only sample injections were performed during the flights. Time series of the local acceleration and temperature of the channel were recorded during the entire flight using LSM9DS0 accelerometer sensors and a TMP36 thermometer sensor, which were all mounted onto an Arduino-based electronic setup made by our team. All of the instruments used were controlled electronically.

\subsection{Image analysis}

The images recorded during the flights were processed using the free image analysis software ImageJ [33]. Due to vibrations induced by the aircraft, the particles were moving too fast to track them using an automated routine; therefore, we tracked them manually, using the Manual Tracking package for ImageJ. The results were filtered using a moving average with an $N=$ 10 block size in order to filter out the high frequency components of the particles' velocity.

\subsection{Conception and fabrication of a set-up adapted to the zero-z flights}

The only way to run zero-gravity experiments, outside the International Space Station is to compensate for the gravitational effect on Earth by freefalling in a controlled parabolic trajectory. At the top of the parabolic trajectory, approximately $22 \mathrm{~s}$ of micro-gravity can be achieved. The microgravity phase is preceded and followed by periods of hyper-gravity, during which vertical acceleration may reach up to $1.8 \mathrm{~g}$ (Fig. 7). The wide range of gravitational forces experienced within the flights allows us to study the influence of both micro and hyper-gravity on various physical phenomena. During a zero-g flight, it is possible to run experiments during a succession of 30 parabola, each 75 seconds long and separated by 105 seconds in standard gravity conditions. There are longer breaks in standard gravity conditions after every fifth parabola.

In order to run experiments in a zero-g flight, one must adapt the setup to two types of constraints: first, constraints imposed by security considerations and specific integration limitations inside the aircraft, and second, 


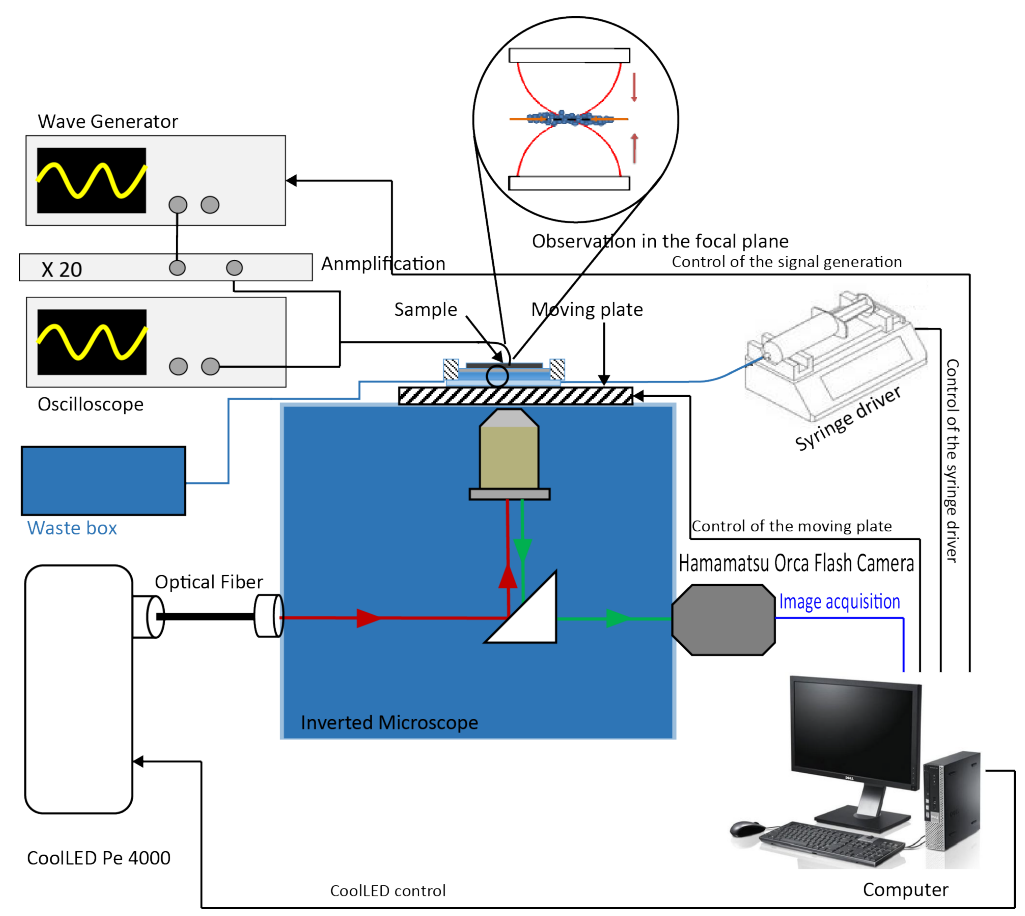

Figure 5: Detailed sketch of the full experimental setup. A computer controls the syringe pump, camera, CoolLED and wave generation.

constraints imposed by the users and the need to control as many parameters as possible in the simplest way. Since the microgravity phase of each experiment can last a maximum of 22 seconds and the standard gravity phase a maximum of 105 seconds, the experimental parameters must be changed quickly between each parabola. Therefore, the experimental setup must be optimized and controlled electronically as much as possible.

Our setup did not exhibit major hazards issues. However, dealing with liquids in microgravity environments is challenging, and in order to prevent leaking, we designed a double confinement system consisting of a fluid closed circuit between the syringe and the waste collection vial within an IP58 Zarges box enclosing the whole experiment. The overall structure was also reinforced to be able to survive up to $7 \mathrm{~g}$ acceleration.

The noisy environment in which we conducted our experiments led to a number of issues, like unwanted flows induced by vertical acceleration variations, temperature elevation thanks to the closed environment, and vibrations. Since we were tracking microparticles with a microscope, even vi- 
(a)

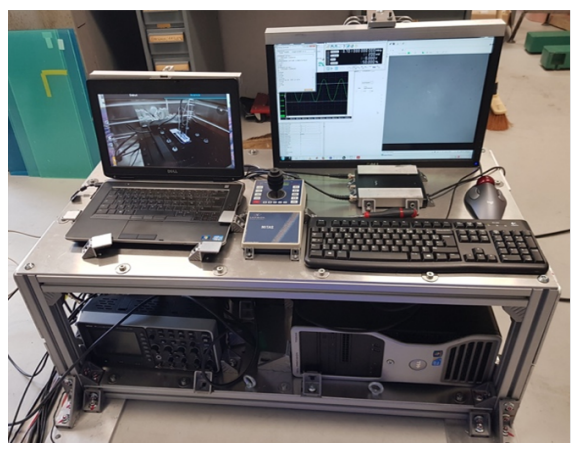

(b)

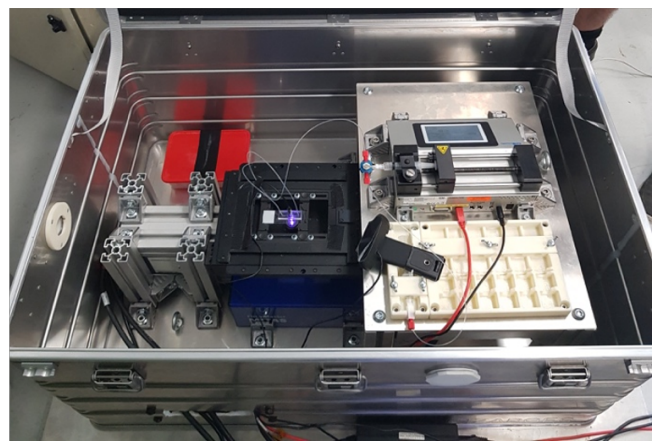

Figure 6: Pictures showing the final setup used on the Airbus Zero-G. (a) Control rack supporting the computers, illumination source and control commands of the actuation devices. (b) Experimental setup, including an inverted microscope, a syringe pump and a Hamamatsu OrcaFlash camera, all integrated into a Zarges box to ensure containment of the fluids during zero-g flights.

brations with a very small amplitude could interfere with our results. Thus, it was very important to limit as much as possible the transfer of vibrations from the aircraft into the box that encased our experiment.

\subsection{Nanorods}

We used the same pure gold nanorods that were used in previous studies [20]. They are approximately $3 \mu \mathrm{m}$ long, with a 0.3-0.4 $\mu \mathrm{m}$ diameter (Fig. 8). They are obtained by electrodeposition of metal ions in the pores of an aluminium oxide filter membrane, growing from a sacrificial silver electrode [34].

\section{Experimental observations in zero gravity con- ditions}

The self-acoustophoresis of nanorods has been studied during each of the three phases of zero-g parabolic flights: the micro-gravity phase $\left(10^{-2} \mathrm{~g}\right)$, the two hyper-gravity phases $(1.8 \mathrm{~g})$, and the standard gravity phases both on the ground and between parabolas. In principle, changing the gravity should also change the axial equilibrium position of the acoustic levitation 
(a)

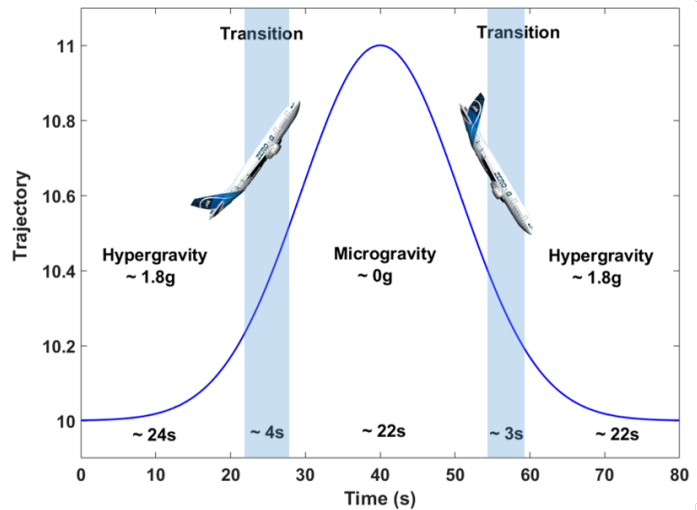

(b)

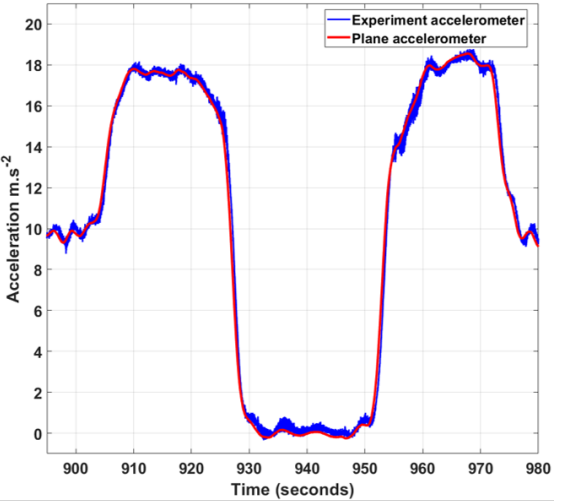

Figure 7: (a) During a daily campaign, the Zero-G Airbus experiences 30 parabolas. Each parabola contains three phases: two hyper-gravity phases and one zero gravity phase. (b) Measurement of the local acceleration on our experiment (blue line) compared with the plane accelerometer (red line). The aircraft accelerometer data is filtered at $7 \mathrm{~Hz}$, while our accelerometer polls up to $400 \mathrm{~Hz}$. We carry out experiments during both the micro-gravity and hyper-gravity phases to evaluate the different gravitational forces influence on the particle aggregates equilibrium positions and spatial organizations.

plane $z_{f o c}$, as illustrated in Fig. 1. The effect of gravity on the equilibrium position depends on the density of the particles or nanorods being used, and should be the greatest for high-density gold or platinum nanorods.

Typical visualizations obtained during the three phases of gravity that occur during zero-g parabolic flights are shown in Fig. 9. All experiments have been carried out with the same sample of gold nanorods and with a given acoustic frequency $\left(f_{a c}=3.6 \mathrm{MHz}\right)$ and acoustic energy density $\left(25 \mathrm{~J}^{-3}{ }^{-3}\right)$. In both standard and hyper-gravity, a large cluster of dense swarming nanorods can be observed (movie online). The nanorods move randomly and autonomously inside the aggregate, and can be seen as bright, elongated objects, because of the reflection of light over the long axes of the metallic rods.

First, we observe that the axial position of the levitation plane indeed changes with gravity. During the experiments, we had to adjust the microscope focus to be able to observe the cluster of nanorods. The axial shift of the levitation plane could be estimated based on the information pro- 


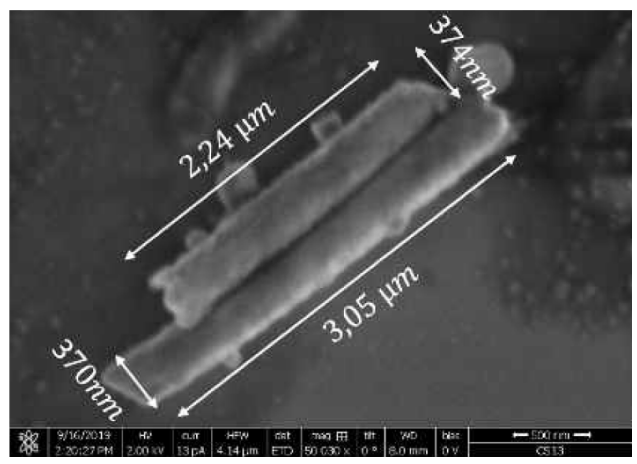

Figure 8: SEM picture of gold nanorods used in these experiments.

vided by the microscope motorized moving stage, and was dependent on the acoustic force applied. The axial position of the levitation plane can change by as much as 20 percent of the channel's total height. For larger deviations we observe a sinking of the cluster. This observation confirms that the levitation plane is not located in the acoustic pressure node, but below it, and that the denser the particles, the lower this position.

Next, we observe that micro-gravity drastically changes the shape and organization of the aggregate of nanorods. In microgravity, unlike in standard and hypergravity, the nanorods that comprise the aggregate are barely visible. They are far less luminous than in non-zero-g conditions, and appear as tiny, dimly illuminated stationary dots. It is important to emphasize that the transition is very sharp, as demonstrated in the video added as supplementary material. The nearly instantaneous change in the spatial organization of nanorods is reminiscent of the nematic transition of liquid crystal. This transition was observed clearly in three successive parabolas.

Our interpretation of these observations is that the nanorods main axes are no longer aligned with the horizontal focusing plane. Indeed, if their long axes rotate quickly to align with the acoustic axial axis, it leads to a strong reduction in the reflection of incident light by the nanorods. Since we are working in reflection (observation perpendicular to the levitation plane), the light reflected toward the camera is also strongly reduced. The rotation and realignment also lead to an apparently less dense aggregate.

This unexpected result challenges the standard hypothesis used until now: why do the nanorods move into the acoustic focusing plane with their main axis parallel to the horizontal $(x, y)$ plane? And, what is the influence of the real location of the levitation plane relative to the location of the acoustic pressure node? These points will be discussed in the following 


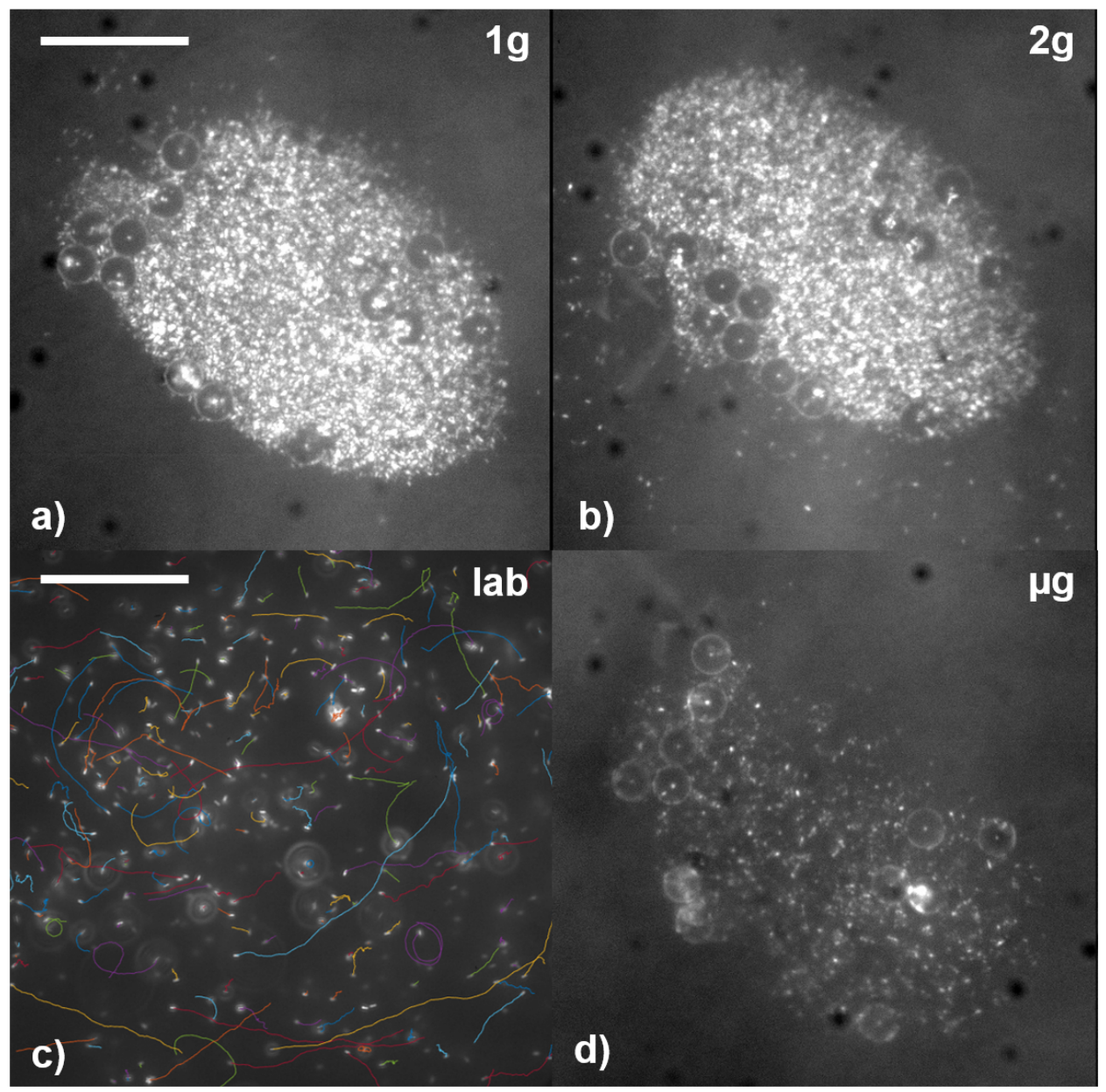

Figure 9: Visualizations of a large aggregate of acoustically-propelled nanorods in acoustic levitation in $1 \mathrm{~g}$ (a), $1.8 \mathrm{~g}$ (b) and micro-gravity (d). The large circles observed in the aggregates are $15 \mu \mathrm{m}$ polystyrene particles that were used to find the equilibrium positions of aggregates prior to the experiment, and were not completely washed away by the buffer. We also show the trajectories of nanorods in laboratory conditions (video online) (c).

section. 


\section{Discussion}

Most of the established knowledge about self-acoustophoresis relies upon experiments conducted in standard gravity conditions, which leads to this question: will the behavior of nanorods change when the local vertical acceleration changes? This question is further justified by the observation that the axial equilibrium position of the levitation plane may be different in non-standard gravity conditions than the position of the acoustic nodal plane, as described in the previous sections. Since all the current theoretical frameworks proposed to explain acoustofluidic propulsion rely on the hypothesis that the nanorods are propelled inside a levitation plane located at the pressure node $[23,24]$, i.e. in a plane where the axial component of the $\mathrm{ARF}$ vanishes and the transverse component is non-null, our observation may have consequences in terms of the actual force fields applied to the nanorods and, subsequently, on their propulsion. However, the models rely only upon the hypothesis that a rod vibrating in a fluid induces streaming, and do not take into account the radiation processes. (Note: the ARF on elongated non-spherical objects is an active area of research [35, 36, 37, 38]). Another standard hypothesis is that the long axis of the rods is always inside the levitation plane, even if this hypothesis is never discussed or justified.

\subsection{Discussion of the orientation of the nanorods main axis relative to the levitation plane}

We have shown in the previous sections that the orientation of nanorods changes in the absence of gravity. From this, we assume that the nanorods axial position within the acoustic field plays a role in the particles response to acoustic actuation.

We first discuss the origin of the orientation of the nanorods main axes in the acoustic focusing plane. Recall that the ARF has two components: an axial component, defined in eq. 1, and a transverse component, $\boldsymbol{F}_{\text {rad }}^{\text {axial }}$. The transverse component is usually negligible relative to the axial component, except in the nodal plane where the axial component vanishes $[39,40]$. The transverse component of the ARF (T-ARF) for a radially symmetric acoustic wave in the nodal plane has been derived by Whitworth [41]:

$$
\boldsymbol{F}_{\text {rad }}^{\text {radial }}(x, y)=8 a^{3} \frac{3\left(\rho_{p}-\rho_{f}\right)}{\rho_{f}+2 \rho_{p}} \nabla\left\langle E_{a c}\right\rangle(x, y)
$$

This component depends directly upon the radial gradient of the acoustic energy $\boldsymbol{\nabla}\left\langle E_{a c}\right\rangle(x, y)$ and is responsible for the aggregation of particles in the 
levitation plane toward the local maximum of acoustic energy in the acoustic focusing plane, which is the final equilibrium position that we observe in standard gravity conditions.

In these experiments, we used a packaged cylindrical transducer which has a relatively well-controlled spatial acoustic energy field. Acoustic measurements conducted on this transducer [42] show that the acoustic energy field is nearly Gaussian with a well-defined maximum in the center. Using the cylindrical symmetry of the acoustic field, we define the spatial dependence of the acoustic energy density following Whitworth and al [12]:

$$
E_{a c}(r, z)=E_{a c}^{0} J_{0}\left(X_{01} \frac{r}{R_{c}}\right)^{2} \sin ^{2}(k z)
$$

where $E_{a c}^{0}$ is the nominal acoustic energy density on the axis of the acoustic transducer, $J_{0}$ is a Bessel function of the first kind, $R_{c}$ is the radius of the cavity, and $X_{01}$ is the first solution to the equation $J_{0}(x)=0$.

This leads to a stable and repeatable positioning of the aggregation area in the center of the cylindrical acoustic force field with spherical particles moving toward the center of cylindrical cavity to form large, stable aggregates. However, the standard expression for the transverse component of the $\mathrm{ARF}$ is incomplete. As previously noted, in standard conditions on Earth, where large aggregates can be quickly formed, gravity causes the real acoustic focusing plane to be lower than the acoustic nodal plane. Therefore, we must use the full expression [12] of the transverse component of the ARF which includes both the $z$ dependency and a second term dealing with the fluid and particles compressibility which is no longer negligible when the particles are not in the nodal plane:

$\boldsymbol{F}_{\text {rad }}^{\text {radial }}(x, y, z)=8 a^{3} \nabla\left\langle E_{a c}\right\rangle(x, y) \times\left[\frac{3\left(\rho_{p}-\rho_{f}\right)}{\rho_{f}+2 \rho_{p}} \sin ^{2}(k z)-\frac{\kappa_{f}-\kappa_{p}}{\kappa_{f}} \cos ^{2}(k z)\right]$

where $\kappa$ is the fluid or particle compressibility. The T-ARF, as shown, depends upon the differences in the density and compressibility of the particles and the surrounding fluid. In our case, $a^{3}$ should be replaced by the volume of the rods.

When dealing with spheres, $\boldsymbol{F}_{\text {rad }}^{\text {axial }}$ leads to the creation of compact aggregates, whereas for elongated particles, like nanorods, the radial forces will first force the objects to change their orientation. Indeed, an elongated object cannot stay horizontal when trapped within a radial force field; any slight movement outside of the horizontal plane will trigger its realignment 


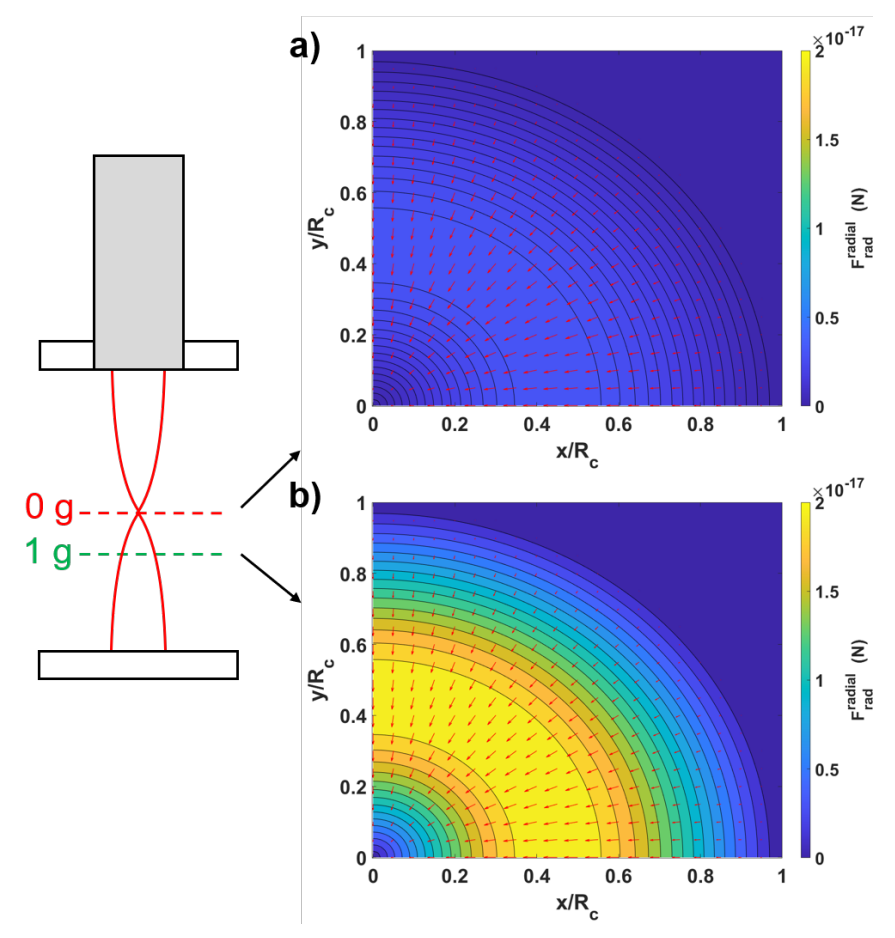

Figure 10: Evolution of the radial component of the ARF for 1 um gold particles in a $3.6 \mathrm{MHz}$ acoustic field with the same geometry as the one used in our experiments. The two figures represent a top view quarter section plotted at mid-height of the resonator (a), exactly in the geometric nodal pressure plane, and in the levitation plane in standard gravity (b). We use the same color scale for both plots to highlight the difference in amplitude of the force along the axis of the resonator.

along the vertical axis. Since nanorods have a very small radius, random Brownian oscillations are sufficient to tilt them around the horizontal axis, leading to a larger radial force while the apparent surface subjected to the radial force increases. During the zero-g phase of parabolic flights, the equilibrium position of the nanorods may be changing sharply in the axial direction, while at the same time maintaining the same equilibrium position (Fig. 11).

In hyper-gravity, on the other hand, the equilibrium position is lower than the nodal plane, and the T-ARF remains strong enough to force the nanorods to gather around the $z$ axis, even as it becomes much weaker than the ARF axial component and gravitational acceleration (Fig. 12). 


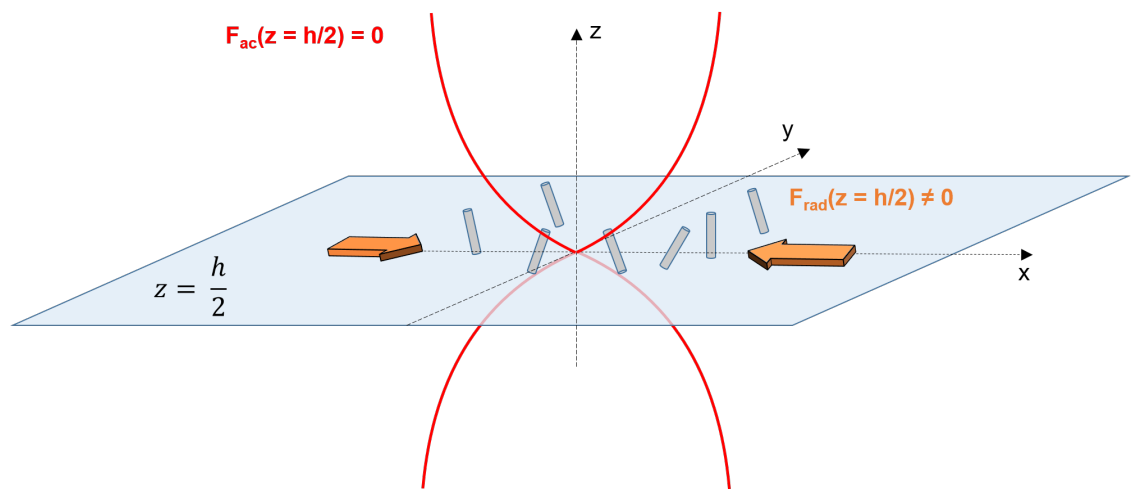

Figure 11: Visualization of the force balance in the nodal plane $(z=h / 2)$ in microgravity. In the nodal plane, the axial component of the ARF is null while the transverse component is not, even if it is weak. The orientation of the nanorods depends only upon the T-ARF which is radially distributed and oriented toward the center of the cavity. A slight movement of the nanorods outside of the nodal plane leads to a reorientation along the $z$ axis.

The equilibrium position of the main axis of the rods is now forced by the balance between gravity and axial ARF, leading to the orientation of the nanorods inside the $(x, y)$ plane. This hypothesis explains the transition observed in the video when the gravity changes from $0 \mathrm{~g}$ to $1.8 \mathrm{~g}$.

\subsection{Breaking up of symmetry as a component to the propul- sion?}

We have shown that microgravity leads to a clear reorganization of the nanorods along the axial direction. Average velocities of gold nanorods are presented in Table 1 for several values of vertical acceleration. We attempted to measure the nanorods propulsion speed during the microgravity phases of the parabolic flights, but their positions were difficult to track due to the low contrast of the images taken during this phase, which we discussed in a previous section. The aircraft's own vibrations also introduced too many uncertainties to precisely quantify the propulsion speeds during the microgravity phases. Future microgravity campaigns with an upgraded experimental setup are planned so that we can obtain more robust data. However, in spite of the noisy environment, we were able to discern that the nanorods move less in microgravity than in standard or hypergravity. This 


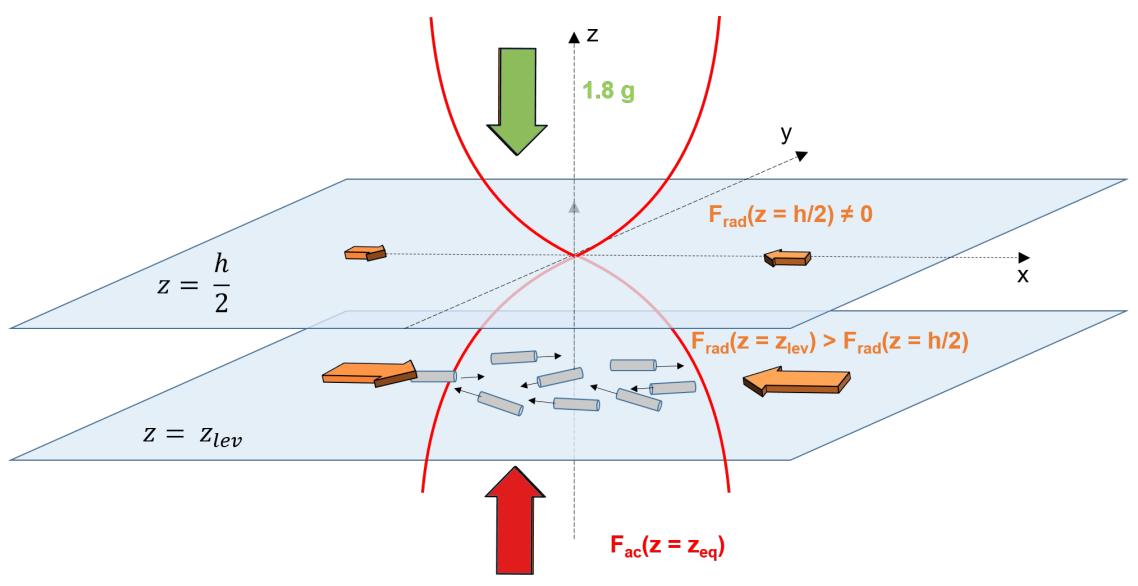

Figure 12: Visualization of the force balance in the acoustic focusing plane $(z<h / 2)$ in hyper-gravity. In the equilibrium plane, the axial component of the ARF is large whereas the transverse component is much weaker. The orientation of the nanorods depends only upon the ARF axial component, which is oriented upward, and on gravity, which is oriented downward. A slight movement of the nanorods outside of the $z$ axis leads to a reorientation into the $(x, y)$ plane.

suggests that the horizontal orientation of the nanorods along the acoustic levitation plane is a key step for self-propulsion. We have shown that in both standard gravity and hypergravity, the orientation of the nanorods is forced into the horizontal plane. Of course, because of Brownian motion for homogeneous rods or because of an asymmetric mass distribution for bi-metallic nanorods, the main axis of the nanorods experience a slight rotation, creating small angles existing between the main axes of the nanorods and the horizontal plane. This slight deviation from the horizontal plane may be a key step in triggering the self-propulsion of the nanorods, since it breaks the axial symmetry.

When the nanorods are tilted at an angle with the $(x, y)$ levitation plane, the T-ARF acts on a larger cross-section of the nanorods which in turn are propelled randomly inside the plane (Fig. 13). This phenomenon is increased for dense, bimetallic nanorods for which the equilibrium orientation is also associated with the mass distribution. The denser end of the rod tilts lower than the lighter end (it sediments faster), leading to a given angle $\alpha$ relative to the levitation plane. This interpretation is compatible with the observations of Ahmed et al. [43] who noticed that the bimetallic nanorods 
are propelled with the lighter end leading first. If these hypotheses are correct, then the T-ARF could be responsible for a part of the nanorods self-propulsion.

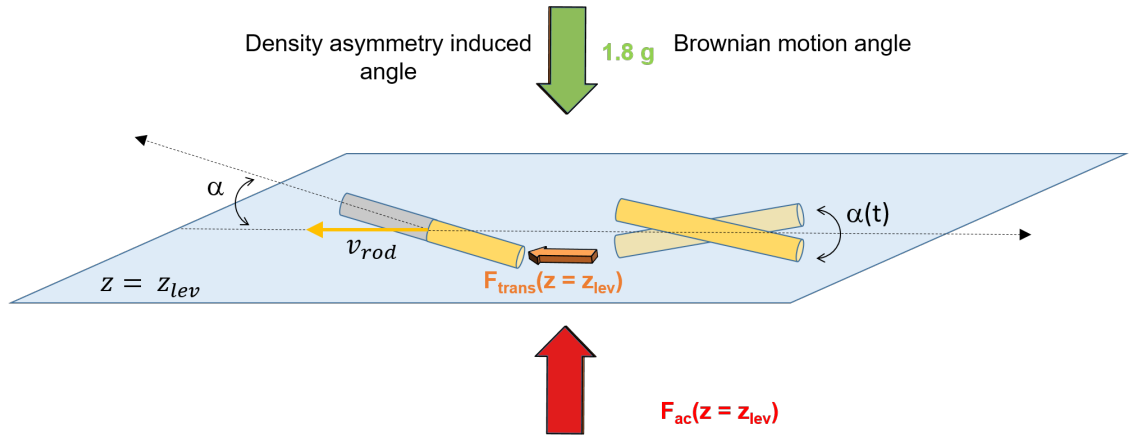

Figure 13: Possible mechanism leading to acoustofluidic propulsion of the nanorods in the acoustic levitation plane $(z<h / 2)$ in hypergravity (and standard gravity). In the equilibrium plane, the axial component of the ARF is large whereas the transverse component is much weaker. The orientation of the nanorods main axes is mainly along the $(x, y)$ plane. Still, we are dealing with dense objects with very small dimensions, with a radius of approximately $0.5 \mathrm{\mu m}$.

Interestingly, Ahmed et al. [43] have shown that lower density nanorods are propelled faster than higher density nanorods, a phenomenon that is not addressed in the hypotheses proposed by Nadal \& Lauga [23] regarding the streaming effect (they are, however, addressed in [24]). If the T-ARF is indeed responsible for the propulsion of the nanorods, then this density dependency of the propulsion velocity follows the model, since the T-ARF depends explicitly upon the rods density (eq. 7). Of course, the effective velocity is simultaneously lowered by the Stokes force, so the velocity is not dependent solely upon the T-ARF. Further, the Stokes force is different for cylinders than for spheres, so the magnitude of its impact varies depending on whether it is acting upon a spherical particle or an elongated rod.

If these hypotheses are true, future experiments regarding the small size and velocity of the nanorods will demonstrate that nanorods will always be slightly tilted. We expect that the denser end of the rod would be slightly out-of-focus, in images capturing their self-propulsion. 


\subsection{Propulsion velocity}

Assuming our hypotheses are valid, it is possible to estimate the velocity of the propulsion caused by the mechanism described in the previous section. To estimate the velocity of elongated particles, such as the nanorods used in our experiments, we must consider the Stokes force for cylinders. For rods with a mean length $L_{r o d}$ and a mean diameter $a_{r o d}$, moving with a velocity $v_{\text {rod }}$ in a fluid at rest, and assuming their long axes are aligned with their velocity, the Stokes force can be defined as [44]:

$$
\boldsymbol{F}_{\text {Stokes }}=\frac{2 \pi \eta L_{\text {rod }} u_{\text {rod }}}{\log \left(L_{\text {rod }} / a_{\text {rod }}\right)-(3 / 2)+\log (2)}=\Gamma \boldsymbol{u}_{\text {rod }}
$$

where $\eta$ is the fluid viscosity. Based on the hypotheses above, we can postulate that the T-ARF plays a role in the propulsion of the nanorods. Starting with the expression derived above, acting on the volume of the nanorod, and writing an equilibrium between the Stokes force and the TARF (approximated for cylinders by assuming a sphere of equivalent volume) leads to the following expression for the propulsion velocity of the rods in the acoustic levitation plane, in $z=z_{\text {lev }}$ :

$$
\begin{aligned}
\boldsymbol{u}_{\text {rod }}\left(x, y, z_{\text {lev }}\right)=\frac{L_{\text {rod }} a_{\text {rod }}^{2}}{\Gamma} \boldsymbol{\nabla}\left\langle E_{\text {ac }}\right\rangle\left(x, y, z_{l e v}\right) \times & \\
& {\left[\frac{3\left(\rho_{p}-\rho_{f}\right)}{\rho_{f}+2 \rho_{p}} \sin ^{2}\left(k z_{l e v}\right)-\frac{\kappa_{f}-\kappa_{p}}{\kappa_{f}} \cos ^{2}\left(k z_{l e v}\right)\right] }
\end{aligned}
$$

However, when this expression is evaluated for the geometrical parameters of the rods used in our experiments, the magnitude of the resulting velocities ranges between $10^{-1}$ and $10^{-3} \mu \mathrm{m} . \mathrm{s}^{-1}$, far less than what we typically measure in the lab, or what we measured during the microgravity campaign (Table 1). This is most likely due to the crude assumption used to express the T-ARF for a cylinder by substituting an expression for sphere of similar volume; the inhomogeneity of the nanorods geometry and mass distribution may account for the amplified force expressed through these higher velocities, and will be explored in future refinements of this model. It is also important to note that the T-ARF is centripetal in our assumptions; if it were the sole cause of the propulsion, we would observe centripetal motion aligned with the force field, which we do not. Nevertheless, we do observe a radial confinement of the self-propelled nanorods, and while this mechanism cannot alone explain the phenomenon of acoustic propulsion, 
it highlights the importance of the $z$ position in the acoustic field on the velocity observed.

\begin{tabular}{lcc}
\hline Local acceleration & Mean velocity & Standard deviation \\
\hline Og $(\mathrm{n}=0)$ & not reliable & not reliable \\
$\lg (\mathrm{n}=311)$ & $28.02 \mu m . s^{-1}$ & $\sigma=4.59$ \\
$2 \mathrm{~g}(\mathrm{n}=31)$ & $54.16 \mu \mathrm{m} . \mathrm{s}^{-1}$ & $\sigma=23.9$ \\
\hline
\end{tabular}

Table 1: Propulsion velocities of gold nanorods measured under similar acoustic conditions for micro, standard and hypergravity. Values for $1 \mathrm{~g}$ were taken from laboratory experiments, with low ambient noise, and values for $0 \mathrm{~g}$ and $2 \mathrm{~g}$ were taken from the zero-g flight experiments, in which ambient noise was high. No accurate quantitative results could be obtained in $0 \mathrm{~g}$; in addition to the high level of ambient noise, rods in zero-g are barely visible due to the orientation of their long axis relative to the horizontal focusing plane.

\section{Conclusion}

The acoustofluidic-propulsion of gold nanorods has been studied in zero-g flights in order to evaluate the influence of gravity, (from hyper-gravity 1.8 $\mathrm{g}$ to microgravity $10^{-2} \mathrm{~g}$ ) on the position of the acoustic levitation plane. In our experiments, we showed that a shift in the gravitational force leads to a variation in the balance between the buoyant and acoustic forces. In spite of the challenges related to running such experiments in zero-g flights, such as the difficulty in tracking individual nanorods in a noisy environment, we gleaned two significant observations that shed new light on the so-called "self-acoustophoresis".

The first major observation is that a cluster of nanorods undergoes a sharp transition when entering into micro-gravity from hypergravity: the previously bright, dense and active cluster suddenly becomes dimmer and less compact. This observation can be explained by a sharp transition in the orientation of rods long axes which switch from horizontal to axial. This reorientation suggests that if the axial forces are lowered relative to the radial forces in the nodal plane, the transverse component of the ARF aligns the rods in the axial direction.

The second notable observation is that nanorods in hypergravity align toward the horizontal plane, and yet still form a large cluster. This clustering proves that the T-ARF in hypergravity is not insignificant, as it is strong 
enough to confine the nanorods in an aggregate. At the same time, the rods realignment along the horizontal plane proves that the balance between gravity and ARF has changed, resulting in the observed new equilibrium orientation of the rods. Our observations also demonstrate that the T-ARF continues to act upon the nanorods in microgravity, forcing them to swith their axes vertically. As the T-ARF is now much weaker than the axial forces in standard and hypergravity, the rods are only slightly tilted out of the horizontal plane and are then pushed forward by the T-ARF, leading to random movement of the nanorods inside the horizontal plane, and confined within the radial acoustic force field.

We also emphasize the fact that the classic assumption that the levitation plane and the geometric pressure node are superposed may be incorrect for dense particles and low-amplitude acoustic fields, and can lead to an underestimation of the T-ARF. We recall the full expression of the T-ARF as proposed by Whitworth et al. [12], and use it, together with a modified expression of the Stokes force adapted to elongated objects, to propose an analytic expression for the propulsion velocity of nanorods. The proposed expression, however, strongly underestimate the propulsion velocity, and requires further refinement.

The hypotheses we propose are compatible with previous results, and with the theory based solely on acoustic streaming. Further research will involve precisely tracking the particles in hyper and micro gravity by improving the experimental setup, further developing the model presented here, and assessing it extensively.

\section{Acknowledgements}

We would like to thank the CNES for funding the experiments and Novespace for their continuous help in the preparation and conception of the setup adapted to the Airbus Zero-G constraints. We also wish to thank the Fondation Bettencourt and FIRE (Frontiers of Innovation in Research and Education) Doctoral School for G. Dumy's PhD grant. We also thank A. Castro and L. Bellebon for their participation to the zero-g flights.

\section{References}

[1] J. M. Fritsch-Yelle, J. B. Charles, M. M. Jones, and M. L. Wood. Microgravity decreases heart rate and arterial pressure in humans. Journal of Applied Physiology, 80(3):910-914, 1996. 
[2] Paul Avan, Hervé Normand, Fabrice Giraudet, Grégory Gerenton, and Pierre Denise. Noninvasive in-ear monitoring of intracranial pressure during microgravity in parabolic flights. Journal of Applied Physiology, 125(2):353-361, 2018.

[3] Shigehiko Ogoh, Michaël Marais, Romain Lericollais, Pierre Denise, Peter B Raven, and Hervé Normand. Interaction between graviception and carotid baroreflex function in humans during parabolic flight-induced microgravity. Journal of Applied Physiology, 125(2):634-641, 2018.

[4] Francine E. Garrett-Bakelman, Manjula Darshi, Stefan J. Green, Ruben C. Gur, Ling Lin, Brandon R. Macias, Miles J. McKenna, Cem Meydan, Tejaswini Mishra, Jad Nasrini, Brian D. Piening, Lindsay F. Rizzardi, Kumar Sharma, Jamila H. Siamwala, Lynn Taylor, Martha Hotz Vitaterna, Maryam Afkarian, Ebrahim Afshinnekoo, Sara Ahadi, Aditya Ambati, Maneesh Arya, Daniela Bezdan, Colin M. Callahan, Songjie Chen, Augustine M. K. Choi, George E. Chlipala, Kévin Contrepois, Marisa Covington, Brian E. Crucian, Immaculata De Vivo, David F. Dinges, Douglas J. Ebert, Jason I. Feinberg, Jorge A. Gandara, Kerry A. George, John Goutsias, George S. Grills, Alan R. Hargens, Martina Heer, Ryan P. Hillary, Andrew N. Hoofnagle, Vivian Y. H. Hook, Garrett Jenkinson, Peng Jiang, Ali Keshavarzian, Steven S. Laurie, Brittany Lee-McMullen, Sarah B. Lumpkins, Matthew MacKay, Mark G. Maienschein-Cline, Ari M. Melnick, Tyler M. Moore, Kiichi Nakahira, Hemal H. Patel, Robert Pietrzyk, Varsha Rao, Rintaro Saito, Denis N. Salins, Jan M. Schilling, Dorothy D. Sears, Caroline K. Sheridan, Michael B. Stenger, Rakel Tryggvadottir, Alexander E. Urban, Tomas Vaisar, Benjamin Van Espen, Jing Zhang, Michael G. Ziegler, Sara R. Zwart, John B. Charles, Craig E. Kundrot, Graham B. I. Scott, Susan M. Bailey, Mathias Basner, Andrew P. Feinberg, Stuart M. C. Lee, Christopher E. Mason, Emmanuel Mignot, Brinda K. Rana, Scott M. Smith, Michael P. Snyder, and Fred W. Turek. The nasa twins study: A multidimensional analysis of a year-long human spaceflight. Science, 364(6436), 2019.

[5] Jeanne L. Becker and Glauco R. Souza. Using space-based investigations to inform cancer research on earth. Nature Reviews Cancer, 13(5):315-327, 2013.

[6] Rajneesh Jha, Qingling Wu, Monalisa Singh, Marcela K. Preininger, Pengcheng Han, Gouliang Ding, Hee Cheol Cho, Hanjoong Jo, Kevin O. 
Maher, Mary B. Wagner, and Chunhui Xu. Simulated microgravity and $3 \mathrm{~d}$ culture enhance induction, viability, proliferation and differentiation of cardiac progenitors from human pluripotent stem cells. Scientific Reports, 6(1):30956, 2016.

[7] Jonathan Baio, Aida F. Martinez, Ivan Silva, Carla V. Hoehn, Stephanie Countryman, Leonard Bailey, Nahidh Hasaniya, Michael J. Pecaut, and Mary Kearns-Jonker. Cardiovascular progenitor cells cultured aboard the international space station exhibit altered developmental and functional properties. npj Microgravity, 4(1):13, 2018.

[8] Daniela Grimm, Markus Wehland, Jessica Pietsch, Ganna Aleshcheva, Petra Wise, Jack van Loon, Claudia Ulbrich, Nils E. Magnusson, Manfred Infanger, and Johann Bauer. Growing tissues in real and simulated microgravity: New methods for tissue engineering. Tissue Engineering Part B: Reviews, 20(6):555-566, 2014.

[9] Victor Camberos, Jonathan Baio, Leonard Bailey, Nahidh Hasaniya, Larry V. Lopez, and Mary Kearns-Jonker. Effects of spaceflight and simulated microgravity on yap1 expression in cardiovascular progenitors: Implications for cell-based repair. International Journal of Molecular Sciences, 20(11), 2019.

[10] Louis Vessot King. On the acoustic radiation pressure on spheres. Proceedings of the Royal Society of London. Series A-Mathematical and Physical Sciences, 147(861):212-240, 1934.

[11] K Yosioka and Y Kawasima. Acoustic radiation pressure on a compressible sphere. Acta Acustica united with Acustica, 5(3):167-173, 1955.

[12] Glenn Whitworth and W. T. Coakley. Particle column formation in a stationary ultrasonic field. The Journal of the Acoustical Society of America, 91(1):79-85, January 1992.

[13] Luz Angelica Castro and Mauricio Hoyos. Determination of the secondary bjerknes force in acoustic resonators on ground and in microgravity conditions. Microgravity Science and Technology, 28(1):11-18, 2016.

[14] Salomé Gutiérrez-Ramos, Mauricio Hoyos, and J. C. Ruiz-Suárez. Induced clustering of escherichia coli by acoustic fields. Scientific Reports, $8(1): 4668,2018$. 
[15] Tatsuki Fushimi, Asier Marzo, Bruce W Drinkwater, and Thomas L Hill. Acoustophoretic volumetric displays using a fast-moving levitated particle. Applied Physics Letters, 115(6):064101, 2019.

[16] Pierre-Yves Gires, Jerome Duplat, Aurelien Drezet, and Cedric Poulain. The acoustic radiation force: a gravitation-like field. arXiv:1805.01634 [physics], May 2018. arXiv: 1805.01634.

[17] Taylor G Wang, AV Anilkumar, CP Lee, and KC Lin. Bifurcation of rotating liquid drops: results from usml-1 experiments in space. 1994.

[18] Robert E Apfel, Yuren Tian, Joseph Jankovsky, Tao Shi, X Chen, R Glynn Holt, Eugene Trinh, Arvid Croonquist, Kathyrn C Thornton, Albert Sacco Jr, et al. Free oscillations and surfactant studies of superdeformed drops in microgravity. Physical review letters, 78(10):1912, 1997.

[19] Philip L Marston, Eugene H Trinh, Jon Depew, and Thomas J Asaki. Oscillatory dynamics of single bubbles and agglomeration in a sound field in microgravity. 1994.

[20] Wei Wang, Luz Angelica Castro, Mauricio Hoyos, and Thomas E Mallouk. Autonomous motion of metallic microrods propelled by ultrasound. ACS nano, 6(7):6122-6132, 2012.

[21] Suzanne Ahmed, Wei Wang, Lamar O. Mair, Robert D. Fraleigh, Sixing Li, Luz Angelica Castro, Mauricio Hoyos, Tony Jun Huang, and Thomas E. Mallouk. Steering Acoustically Propelled Nanowire Motors toward Cells in a Biologically Compatible Environment Using Magnetic Fields. Langmuir, 29(52):16113-16118, December 2013.

[22] Rachel R Collino, Tyler R Ray, Rachel C Fleming, James D Cornell, Brett G Compton, and Matthew R Begley. Deposition of ordered twophase materials using microfluidic print nozzles with acoustic focusing. Extreme Mechanics Letters, 8:96-106, 2016.

[23] François Nadal and Eric Lauga. Asymmetric steady streaming as a mechanism for acoustic propulsion of rigid bodies. Physics of Fluids, 26(8):082001, 2014.

[24] Jesse F. Collis, Debadi Chakraborty, and John E. Sader. Autonomous propulsion of nanorods trapped in an acoustic field. Journal of Fluid Mechanics, 825:29-48, August 2017. 
[25] Nicolas Bertin, Tamsin A Spelman, Olivier Stephan, Laetitia Gredy, Michel Bouriau, Eric Lauga, and Philippe Marmottant. Propulsion of bubble-based acoustic microswimmers. Physical Review Applied, 4(6):064012, 2015.

[26] Jean-François Louf, Nicolas Bertin, Benjamin Dollet, Olivier Stephan, and Philippe Marmottant. Hovering microswimmers exhibit ultrafast motion to navigate under acoustic forces. Advanced Materials Interfaces, 5(16):1800425, 2018.

[27] L. P. Gor'kov. On the forces acting on a small particle in an acoustical field in an ideal fluid. Soviet Physics - Doklady, pages 773-775, 1962.

[28] Alexander A. Doinikov. Acoustic radiation pressure on a compressible sphere in a viscous fluid. Journal of Fluid Mechanics, 267(-1):1, May 1994.

[29] Mikkel Settnes and Henrik Bruus. Forces acting on a small particle in an acoustical field in a viscous fluid. Physical Review E, 85(1):016327, 2012 .

[30] Daniel Ahmed, Thierry Baasch, Bumjin Jang, Salvador Pane, Jürg Dual, and Bradley J. Nelson. Artificial Swimmers Propelled by Acoustically Activated Flagella. Nano Letters, 16(8):4968-4974, August 2016.

[31] Chao Zhou, Leilei Zhao, Mengshi Wei, and Wei Wang. Twists and Turns of Orbiting and Spinning Metallic Microparticles Powered by Megahertz Ultrasound. ACS Nano, 11(12):12668-12676, December 2017.

[32] Syeda Sabrina, Mykola Tasinkevych, Suzanne Ahmed, Allan M Brooks, Monica Olvera de la Cruz, Thomas E Mallouk, and Kyle JM Bishop. Shape-directed microspinners powered by ultrasound. ACS nano, 12(3):2939-2947, 2018.

[33] Caroline A Schneider, Wayne S Rasband, and Kevin W Eliceiri. Nih image to imagej: 25 years of image analysis. Nature methods, 9(7):671, 2012 .

[34] Yang Wang, Rose M. Hernandez, David J. Bartlett, Julia M. Bingham, Timothy R. Kline, Ayusman Sen, and Thomas E. Mallouk. Bipolar Electrochemical Mechanism for the Propulsion of Catalytic Nanomotors in Hydrogen Peroxide Solutions ${ }^{\dagger}$. Langmuir, 22(25):10451-10456, December 2006. 
[35] Philip L Marston, Wei Wei, and David B Thiessen. Acoustic radiation force on elliptical cylinders and spheroidal objects in low frequency standing waves. In AIP Conference Proceedings, volume 838, pages 495-499. AIP, 2006.

[36] FG Mitri. Acoustic radiation force on oblate and prolate spheroids in bessel beams. Wave Motion, 57:231-238, 2015.

[37] Felix Bob Wijaya and Kian-Meng Lim. Numerical calculation of acoustic radiation force and torque acting on rigid non-spherical particles. Acta Acustica united with Acustica, 101(3):531-542, 2015.

[38] Glauber T. Silva and Bruce W. Drinkwater. Acoustic radiation force exerted on a small spheroidal rigid particle by a beam of arbitrary wavefront: Examples of traveling and standing plane waves. The Journal of the Acoustical Society of America, 144(5):EL453-EL459, November 2018.

[39] Toru Tuziuti, Teruyuki Kozuka, and Hideto Mitome. Measurement of Distribution of Acoustic Radiation Force Perpendicular to Sound Beam Axis. Japanese Journal of Applied Physics, 38(Part 1, No. 5B):32973301, May 1999.

[40] Steven M. Woodside, Bruce D. Bowen, and James M. Piret. Measurement of ultrasonic forces for particle-liquid separations. AIChE Journal, 43(7):1727-1736, July 1997.

[41] G Whitworth, MA Grundy, and WT Coakley. Transport and harvesting of suspended particles using modulated ultrasound. Ultrasonics, 29(6):439-444, 1991.

[42] Olivier Dron. Micro-manipulation acoustique de particules: application aux mesures par micro-PIV. PhD thesis, Paris 7, 2011.

[43] Suzanne Ahmed, Wei Wang, Lanjun Bai, Dillon T. Gentekos, Mauricio Hoyos, and Thomas E. Mallouk. Density and Shape Effects in the Acoustic Propulsion of Bimetallic Nanorod Motors. ACS Nano, 10(4):4763-4769, April 2016.

[44] GK Batchelor. Slender-body theory for particles of arbitrary crosssection in stokes flow. Journal of Fluid Mechanics, 44(3):419-440, 1970. 